\title{
SYMMETRIC INVERSE GENERALIZED EIGENVALUE PROBLEM WITH SUBMATRIX CONSTRAINTS IN STRUCTURAL DYNAMIC MODEL UPDATING*
}

\author{
MEIXIANG ZHAO ${ }^{\dagger}$, ZHIGANG JIA ZAND MUSHENG WEI $^{\S}$
}

\begin{abstract}
In this literature, the symmetric inverse generalized eigenvalue problem with submatrix constraints and its corresponding optimal approximation problem are studied. A necessary and sufficient condition for solvability is derived, and when solvable, the general solutions are presented.
\end{abstract}

Key words. Symmetric inverse generalized eigenvalue problem, Submatrix constraint, Optimal approximation.

AMS subject classifications. 15A15, 15A29.

1. Introduction. Let $\mathbb{R}^{m \times n}$ denote the set of all $m \times n$ real matrices, $\mathbb{S R}^{n \times n}$ the set of all $n \times n$ real symmetric matrices, and $\mathbb{C}^{n}$ the $n$-dimensional complex vector space. The dynamic analysis of a mechanical or civil structure by the finite element technique can be modelled by a generalized eigenvalue problem

$$
K_{a} x=\lambda M_{a} x,
$$

where $K_{a}, M_{a} \in \mathbb{S R}^{n \times n}$ are the analytical stiffness matrix and mass matrix, respectively, and $\lambda \in \mathbb{C}$ and $x \in \mathbb{C}^{n}$ are the generalized eigenvalue and corresponding generalized eigenvector. It's well known that the natural frequencies and mode shapes of such a finite element model and ones experimentally measured by a vibration test do not match very well ([4], [20]). The finite element model updating problem is to determine how to update the model to closely match the experimental model data that gives an incomplete set of eigenpairs.

Let $x_{1}, \ldots, x_{p} \in \mathbb{C}^{n}$ be the measured modal vectors and $\lambda_{1}, \ldots, \lambda_{p} \in \mathbb{C}$ be the

${ }^{*}$ Received by the editors on July 30, 2010. Accepted for publication on June 27, 2011. Handling Editor: Bryan Shader.

$\dagger^{\dagger}$ Kewen Institute, Xuzhou Normal University, Jiangsu 221116, P.R. China. Supported by the Natural Science Foundation of Shandong Province under grant ZR2010AM014.

${ }^{\ddagger}$ Department of Mathematics, Xuzhou Normal University, Jiangsu 221116, P.R. China (zhgjia@xznu.edu.cn). Supported by the Universities Natural Science Foundation of Jiangsu Province under grant 10KJD110005, the NSFC under grant 11001144, and the Natural Science Foundation of Jiangsu Province under grant BK2010174.

${ }^{\S}$ College of Mathematics and Science, Shanghai Normal University, Scientific Computing Key Laboratory of Shanghai Universities, Shanghai 200234, P.R. China. Supported by the NSFC under grant 10771073, and the Shanghai Leading Academic Discipline Project under grant S30405. 
measured natural frequencies, where $p \leq n$. The measured mode shapes and frequencies are assumed to be correct, and the mass matrix $M \in \mathbb{S R}^{n \times n}$ and stiffness matrix $K \in \mathbb{S R}^{n \times n}$ to be constructed have to satisfy the dynamic equation

$$
K x_{i}=M x_{i} \lambda_{i}, \quad i=1, \ldots, p
$$

(see e.g. [1], [2], [6]-[8], [11], [12], [16], [17], [22]). Since we are only interested in real matrices, the prescribed eigenpairs must be closed under complex conjugation. Without causing ambiguity, the $p$ prescribed eigenpairs are represented by the matrix form $(X, \Lambda)$, where each block of $\Lambda \in \mathbb{R}^{p \times p}$ is either a 1-by-1 matrix or a 2-by-2 matrix whose eigenvalues are a complex conjugate pair, and $X \in \mathbb{R}^{n \times p}$ represents the "eigenvector matrix" in the sense that each pair of column vectors associated with a 2 -by-2 block in $\Lambda$ retains the real and the imaginary part, respectively, of the original complex eigenvector. Then (1.2) is equivalent to $K X=M X \Lambda$. As model errors can be localized by sensitivity analysis ([10], [19]), residual force approach ([9]), least-squares approach ([13]) and assigned eigenstructure ([5]), it is a usual practice to adjust partial elements of the analytical mass and stiffness matrices. Mathematically, such a partially updating problem can be described as the following two problems:

Problem 1.1. Given $p$ eigenpairs $(X, \Lambda)$, where $X \in \mathbb{R}^{n \times p}$ and $\Lambda \in \mathbb{R}^{p \times p}$ are described as the above and $K_{0}, M_{0} \in \mathbb{S R}^{r \times r}, 1 \leq p, r \leq n, p+r \leq n$, find real matrices $K, M \in \mathbb{S R}^{n \times n}$ such that

$$
K X=M X \Lambda, K([1, r])=K_{0}, M([1, r])=M_{0}
$$

where $K([1, r])$ and $M([1, r])$ are the $r \times r$ leading principal submatrices of $K$ and $M$, respectively.

Problem 1.2. Given $K_{a}, M_{a} \in \mathbb{S R}^{n \times n}$ with $K_{a}([1, r])=K_{0}, M_{a}([1, r])=M_{0}$, find $\hat{K}, \hat{M} \in S_{E}$ such that

$$
\left\|\left(K_{a}, M_{a}\right)-(K, M)\right\|=\inf _{(\hat{K}, \hat{M}) \in S_{E}}\left\|\left(K_{a}, M_{a}\right)-(\hat{K}, \hat{M})\right\|,
$$

where $S_{E}$ is the solution set of Problem 1.1. Here $\|\cdot\|$ denotes the Frobenius norm.

The second problem is to find the best approximation for a given symmetric matrix pencil under a given spectral constraint and a symmetric submatrix pencil constraint. Such a problem always arises in structural dynamic model updating.

Y. Yuan and H. Dai [21] solved the above two problems, where $K_{a}, K_{0}, K$, $M_{a}, M_{0}$, and $M$ are free from the symmetry constraint conditions. In this paper, we consider the symmetric cases in the dynamic analysis of a mechanical or civil structure by the finite element technique. Only by using the Moore-Penrose generalized inverse and the singular value decomposition, we present the solvability condition and the 
expression for the solutions of Problems 1.1 and 1.2. For a special case, we derive a formula of the general solution which is inexpensive to compute and can be used routinely in practice.

Throughout this paper $O(n)$ stands for the set of all $n \times n$ orthogonal matrices; $A^{T}$ the transpose of a real matrix $A ; A^{\dagger}$ the Moore-Penrose generalized inverse of $A$; $P_{A}=A A^{\dagger}, P_{A}^{\perp}=I-P_{A} ; \operatorname{tr}(A)$ the trace of the matrix $A \in \mathbb{R}^{n \times n}$; and $\operatorname{rank}(A)$ the rank of $A$. For $A, B \in \mathbb{R}^{m \times n},(A, B)=\operatorname{tr}\left(B^{T} A\right)$ denotes an inner product in $\mathbb{R}^{m \times n}$.

2. General solutions of Problems 1.1 and 1.2. To facilitate the following discussion, we first make some notations. Let $X \in \mathbb{R}^{n \times p}, K, M \in \mathbb{S R}^{n \times n}$ have the following partitions:

$$
X=\left(\begin{array}{l}
X_{1} \\
X_{2}
\end{array}\right), \quad K=\left(\begin{array}{ll}
K_{0} & K_{1} \\
K_{1}^{T} & K_{2}
\end{array}\right), \quad M=\left(\begin{array}{cc}
M_{0} & M_{1} \\
M_{1}^{T} & M_{2}
\end{array}\right),
$$

where $X_{1}$ and $X_{2}$ have $r$ and $n-r$ rows respectively, $K_{0}, M_{0} \in \mathbb{R}^{r \times r}, K_{1}, M_{1} \in$ $\mathbb{R}^{r \times(n-r)}$, and $K_{2}, M_{2} \in \mathbb{S R}^{(n-r) \times(n-r)}$. Then $K X=M X \Lambda$ can be rewritten as

$$
\left(\begin{array}{ll}
K_{0} & K_{1} \\
K_{1}^{T} & K_{2}
\end{array}\right)\left(\begin{array}{l}
X_{1} \\
X_{2}
\end{array}\right)=\left(\begin{array}{cc}
M_{0} & M_{1} \\
M_{1}^{T} & M_{2}
\end{array}\right)\left(\begin{array}{l}
X_{1} \\
X_{2}
\end{array}\right) \Lambda .
$$

That implies that (1.3) is equivalent to

$$
\begin{aligned}
K_{1} X_{2} & =M_{0} X_{1} \Lambda-K_{0} X_{1}+M_{1} X_{2} \Lambda, \\
K_{1}^{T} X_{1} & =M_{1}^{T} X_{1} \Lambda-K_{2} X_{2}+M_{2} X_{2} \Lambda .
\end{aligned}
$$

Problem 1.1 can be solved by computing the solutions $K_{1}, M_{1}, K_{2}$ and $M_{2}$ of $(2.3)$ and (2.4).

Suppose that the singular value decomposition of $X_{2}$ has been computed

$$
X_{2}=U\left(\begin{array}{cc}
\Sigma & 0 \\
0 & 0
\end{array}\right) V^{T}
$$

where $U=\left[U_{1}, U_{2}\right] \in O(n-r), V=\left[V_{1}, V_{2}\right] \in O(p), \Sigma=\operatorname{diag}\left(\sigma_{1}, \ldots, \sigma_{s}\right), \sigma_{i}>0$, $i=1, \ldots, s, s=\operatorname{rank}\left(X_{2}\right), U_{1} \in \mathbb{R}^{(n-r) \times s}, V_{1} \in \mathbb{R}^{p \times s}$. Similarly, suppose that the following singular value decompositions have been computed

$$
\begin{aligned}
X_{2} \Lambda V_{2} & =\left[P_{1}, P_{2}\right]\left(\begin{array}{cc}
\Omega & 0 \\
0 & 0
\end{array}\right)\left[Q_{1}, Q_{2}\right]^{T}, \\
X_{1} V_{2} Q_{2} & =\left[\tilde{P}_{1}, \tilde{P}_{2}\right]\left(\begin{array}{cc}
\tilde{\Omega} & 0 \\
0 & 0
\end{array}\right)\left[\tilde{Q}_{1}, \tilde{Q}_{2}\right]^{T}, \\
\left(X_{2} \Lambda X_{2}^{\dagger}\right)^{T} & =\left[\hat{U}_{1}, \hat{U}_{2}\right]\left(\begin{array}{cc}
\hat{\Sigma} & 0 \\
0 & 0
\end{array}\right)\left[\hat{V}_{1}, \hat{V}_{2}\right]^{T} .
\end{aligned}
$$


Now we recall some known results, which will be used repeatedly in this paper.

Lemma 2.1. [14] Let $Y, B \in \mathbb{R}^{m \times k}, m \geq k$, be given. Then $A Y=B$ has a symmetric solution $A \in \mathbb{S R}^{m \times m}$ if and only if $B P_{Y^{T}}=B$ and $\left(P_{Y} B Y^{\dagger}\right)^{T}=P_{Y} B Y^{\dagger}$. Moreover, the solution set is

$$
S=\left\{B Y^{\dagger}+\left(B Y^{\dagger}\right)^{T} P_{Y}^{\perp}+P_{Y}^{\perp} H P_{Y}^{\perp}: H \in \mathbb{S R}^{m \times m}\right\},
$$

and the minimal solution under the Frobenius norm is

$$
A_{o p t}=B Y^{\dagger}+\left(B Y^{\dagger}\right)^{T} P_{Y}^{\perp} .
$$

LeMmA 2.2. [3] If $E \in \mathbb{R}^{m \times n}, F \in \mathbb{R}^{p \times q}$ and $G \in \mathbb{R}^{m \times q}$,

$$
E X F=G
$$

has a solution $X \in \mathbb{R}^{p \times q}$ if and only if

$$
E E^{\dagger} G F^{\dagger} F=G
$$

in which case, the general solution of (2.9) can be expressed as

$$
X=E^{\dagger} G F^{\dagger}+Y-E^{\dagger} E Y F F^{\dagger}
$$

where $Y \in \mathbb{R}^{n \times p}$ is an arbitrary matrix.

The next lemma is extracted from the proof of Theorem 1 in [21].

LEMma 2.3. [21] Equation $K_{0} X_{1}+K_{1} X_{2}=M_{0} X_{1} \Lambda+M_{1} X_{2} \Lambda$ with respect to matrices $K_{1}$ and $M_{1}$ is solvable if and only if

$$
K_{0} X_{1} V_{2} Q_{2}=M_{0} X_{1} \Lambda V_{2} Q_{2},
$$

in which case, we have

$$
\begin{aligned}
& K_{1}=K_{10}+L P_{2}^{T} X_{2} \Lambda X_{2}^{\dagger}+W U_{2}^{T}, \\
& M_{1}=M_{10}+L P_{2}^{T},
\end{aligned}
$$

where

$$
\begin{aligned}
M_{10} & =\left(K_{0} X_{1} V_{2}-M_{0} X_{1} \Lambda V_{2}\right)\left(X_{2} \Lambda V_{2}\right)^{\dagger}, \\
K_{10} & =\left[M_{0} X_{1} \Lambda-K_{0} X_{1}+M_{10} X_{2} \Lambda\right] X_{2}^{\dagger},
\end{aligned}
$$

and $L \in \mathbb{R}^{r \times(n-r-t)}, W \in \mathbb{R}^{r \times(n-r-s)}$ are arbitrary matrices. 
As we discussed above, the general solution of Problem 1.1 can be derived by solving (2.3) and (2.4). Lemma 2.3 has presented the solvability condition and the general solution (if exists) of (2.3). Now we study (2.4). Define $\tilde{L}=P_{2} L^{T}, \tilde{W}=$ $U_{2} W^{T}$, and

$$
\Psi=M_{10}^{T} X_{1} \Lambda-K_{10}^{T} X_{1}+\tilde{L} X_{1} \Lambda-\left(X_{2} \Lambda X_{2}^{\dagger}\right)^{T} \tilde{L} X_{1}
$$

Substitute (2.11) and (2.12) into (2.4), to get

$$
K_{2} X_{2}=\Psi-\tilde{W} X_{1}+M_{2} X_{2} \Lambda
$$

with respect to unknown real symmetric matrices $K_{2}$ and $M_{2}$. Repeatedly applying Lemma 2.1 and equations (2.5)-(2.8), and (2.13) has a symmetric solution $K_{2}$ if and only if

$$
\begin{gathered}
M_{2} X_{2} \Lambda V_{2}=\left[-\Psi+\tilde{W} X_{1}\right] V_{2}, \\
U_{1} U_{1}^{T}\left[\Psi+M_{2} X_{2} \Lambda\right] X_{2}^{\dagger} \in \mathbb{S R}^{(n-r) \times(n-r)} .
\end{gathered}
$$

Equation (2.14) has a symmetric solution $M_{2}$ if and only if

$$
\begin{gathered}
\left(X_{2} \Lambda X_{2}^{\dagger}\right)^{T} \tilde{L} X_{1} V_{2} Q_{2}=-\left(K_{10}^{T}+\tilde{W}\right) X_{1} V_{2} Q_{2}, \\
P_{1} P_{1}^{T}\left[-\Psi+\tilde{W} X_{1}\right] V_{2}\left(X_{1} \Lambda V_{2}\right)^{\dagger} \in \mathbb{S R}^{r \times r} .
\end{gathered}
$$

Now we prove that (2.16) always holds. Lemma 2.2 will be repeatedly used without being mentioned in the following analysis. Equation (2.16) has a solution $\tilde{L}$ if and only if

$$
\left[I-\left(X_{2} \Lambda X_{2}^{\dagger}\right)^{T}\left(\left(X_{2} \Lambda X_{2}^{\dagger}\right)^{T}\right)^{\dagger}\right]\left(K_{10}^{T}+\tilde{W}\right) X_{1} V_{2} Q_{2}=0
$$

Recalling the SVD of $\left(X_{2} \Lambda X_{2}^{\dagger}\right)^{T}$ as in (2.8), (2.18) can be rewritten as

$$
\hat{U}_{2}^{T} \tilde{W} X_{1} V_{2} Q_{2}=-\hat{U}_{2}^{T} K_{10}^{T} X_{1} V_{2} Q_{2} .
$$

We can see that (2.19) is always solvable and the expression of its general solution is (2.20) $\tilde{W}=-\hat{U}_{2} \hat{U}_{2}^{T} K_{10}^{T}\left(X_{1} V_{2} Q_{2}\right)\left(X_{1} V_{2} Q_{2}\right)^{\dagger}+\hat{Y}-\hat{U}_{2} \hat{U}_{2}^{T} \hat{Y}\left(X_{1} V_{2} Q_{2}\right)\left(X_{1} V_{2} Q_{2}\right)^{\dagger}$, where $\hat{Y} \in \mathbb{R}^{(n-r) \times r}$ is arbitrary. That means (2.18) always holds and consequently there must exist a matrix $\tilde{L}$ satisfying (2.16). Now substitute (2.20) into (2.16), to get

$$
\left(X_{2} \Lambda X_{2}^{\dagger}\right)^{T} \tilde{L} X_{1} V_{2} Q_{2}=-\hat{U}_{1} \hat{U}_{1}^{T}\left(K_{10}^{T}-\hat{Y}\right) X_{1} V_{2} Q_{2}
$$


The general solution of (2.21) has the form

$$
\tilde{L}=-\left(\left(X_{2} \Lambda X_{2}^{\dagger}\right)^{T}\right)^{\dagger}\left(K_{10}^{T}-\hat{Y}\right)\left(\tilde{P}_{1} \tilde{P}_{1}^{T}\right)+\tilde{Y}-\hat{V}_{1} \hat{V}_{1}^{T} \tilde{Y}\left(\tilde{P}_{1} \tilde{P}_{1}^{T}\right),
$$

where $\tilde{Y}$ is arbitrary. So that (2.16) is always solvable and its general solution can be expressed as in (2.22).

By Lemma 2.1, we can conclude that (2.14) is solvable when (2.16) and (2.17) hold, and its general solution is

(2.23) $M_{2}=B_{M_{2}}\left(X_{2} \Lambda V_{2}\right)^{\dagger}+\left(B_{M_{2}}\left(X_{2} \Lambda V_{2}\right)^{\dagger}\right)^{T} P_{\left(X_{2} \Lambda V_{2}\right)}^{\perp}+P_{\left(X_{2} \Lambda V_{2}\right)}^{\perp} H_{M_{2}} P_{\left(X_{2} \Lambda V_{2}\right)}^{\perp}$

in which $B_{M_{2}}=\left[-\Psi+\left(X_{2} \Lambda X_{2}^{\dagger}\right)^{T} \tilde{L} X_{1}+\tilde{W} X_{1}\right] V_{2}$ and $H_{M_{2}} \in \mathbb{S R}^{(n-r) \times(n-r)}$ is arbitrary. Similarly, if (2.14) and (2.15) hold, then (2.13) has a symmetric solution

$$
K_{2}=B_{K_{2}} X_{2}^{\dagger}+\left(B_{K_{2}} X_{2}^{\dagger}\right)^{T} P_{X_{2}}^{\perp}+P_{X_{2}}^{\perp} H_{K_{2}} P_{X_{2}}^{\perp},
$$

where $B_{K_{2}}=\Psi-\left(X_{2} \Lambda X_{2}^{\dagger}\right)^{T} \tilde{L} X_{1}-\tilde{W} X_{1}+M_{2} X_{2} \Lambda$ and $H_{K_{2}} \in \mathbb{S R}^{(n-r) \times(n-r)}$ is arbitrary.

Now we suppose that there exist $\hat{Y}, \tilde{Y}$ and $H_{M_{2}}$ such that the symmetric conditions (2.15) and (2.17) hold after substituting (2.20), (2.22), and (2.23) into them. Then we can conclude that Problem 1.1 is solvable from the above analysis.

TheOrem 2.4. Problem 1.1 is solvable if and only if (2.10), (2.15) and (2.17) hold. In this case, the general solution is

$$
(K, M)=\left(\begin{array}{llll}
K_{0} & K_{1} & M_{0} & M_{1} \\
K_{1}^{T} & K_{2} & M_{1}^{T} & M_{2}
\end{array}\right)
$$

where $K_{1}, M_{1}, M_{2}, K_{2}$ are given by (2.11), (2.12), (2.23), and (2.24) in which $\hat{Y}$, $\tilde{Y}$, and $H_{M_{2}}$ satisfy (2.15) and (2.17).

Now we study Problem 1.2 under the condition that the solution set $S_{E}$ of Problem 1.1 is not empty. Firstly, recall an useful result in [15].

Lemma 2.5. [15] Suppose that $A \in \mathbb{R}^{q \times m}, \Delta \in \mathbb{R}^{q \times q}$, and $\Gamma \in \mathbb{R}^{m \times m}$, where $\Delta^{2}=\Delta=\Delta^{T}$ and $\Gamma^{2}=\Gamma=\Gamma^{T}$. Then

$$
\|A-\Delta D \Gamma\|=\min _{E \in \mathbb{R}^{q \times m}}\|A-\Delta E \Gamma\|
$$

if and only if $\Delta(A-D) \Gamma=0$, in which case,

$$
\|A-\Delta D \Gamma\|=\|A-\Delta A \Gamma\| .
$$


For given matrices $K_{a} \in \mathbb{R}^{n \times n}$ and $M_{a} \in \mathbb{R}^{n \times n}$ in Problem 1.2, define

$$
K_{a}=\left(\begin{array}{cc}
K_{0} & K_{1}^{(a)} \\
\left(K_{1}^{(a)}\right)^{T} & K_{2}^{(a)}
\end{array}\right), \quad M_{a}=\left(\begin{array}{cc}
M_{0} & M_{1}^{(a)} \\
\left(M_{1}^{(a)}\right)^{T} & M_{2}^{(a)}
\end{array}\right)
$$

where $K_{1}^{(a)}, M_{1}^{(a)} \in \mathbb{R}^{r \times(n-r)}$, and $K_{2}^{(a)}, M_{2}^{(a)} \in \mathbb{S R}^{(n-r) \times(n-r)}$. Given two matrices $C, D$ and a matrix set $S$, the notation $\|C-A\|^{2}+\|D-B\|^{2}=$ min means that $\|C-A\|^{2}+\|D-B\|^{2}$ is minimized by $(A, B) \in S$; i.e.,

$$
\|C-A\|^{2}+\|D-B\|^{2}=\min _{(\hat{A}, \hat{B}) \in S}\left(\|C-\hat{A}\|^{2}+\|D-\hat{B}\|^{2}\right) .
$$

THEOREM 2.6. Under the conditions of Theorem 2.4 and assuming that $\hat{Y}, \tilde{Y}$ and $H_{M_{2}}$ are unique solutions of (2.15) and (2.17), Problem 1.2 has a unique solution

$$
(K, M)=\left(\begin{array}{cccc}
K_{0} & K_{1} & M_{0} & M_{1} \\
K_{1}^{T} & K_{2} & M_{1}^{T} & M_{2}
\end{array}\right)
$$

where $K_{1}, M_{1}, M_{2}, K_{2}$ are given by (2.11), (2.12), (2.23), and (2.24), and $H_{K_{2}}$ and $H_{M_{2}}$ satisfy $P_{X_{2}}^{\perp}\left(K_{2}^{(a)}-H_{K_{2}}\right) P_{X_{2}}^{\perp}=0$ and $P_{\left(X_{2} \Lambda V_{2}\right)}^{\perp}\left(M_{2}^{(a)}-H_{M_{2}}\right) P_{\left(X_{2} \Lambda V_{2}\right)}^{\perp}=0$, respectively.

Proof. Equation (1.4) is equivalent to

$$
\left\|K_{a}-K\right\|^{2}+\left\|M_{a}-M\right\|^{2}=\min .
$$

With the partitions (2.1) and (2.25) in mind, we have

$$
\begin{aligned}
\left\|K_{a}-K\right\|^{2}+\left\|M_{a}-M\right\|^{2}= & 2\left\|K_{1}^{(a)}-K_{1}\right\|^{2}+\left\|K_{2}^{(a)}-K_{2}\right\|^{2}+2 \| M_{1}^{(a)} \\
& -M_{1}\left\|^{2}+\right\| M_{2}^{(a)}-M_{2} \|^{2} .
\end{aligned}
$$

Then (2.26) holds if and only if

$$
\left\|K_{2}^{(a)}-K_{2}\right\|^{2}+\left\|M_{2}^{(a)}-M_{2}\right\|^{2}=\min .
$$

By Lemma 2.5, (2.27) holds if and only if

$$
U_{2} U_{2}^{T}\left(K_{2}^{(a)}-H_{K_{2}}\right) U_{2} U_{2}^{T}=0, P_{2} P_{2}^{T}\left(M_{2}^{(a)}-H_{M_{2}}\right) P_{2} P_{2}^{T}=0 .
$$

REMARK 2.7. We have solved Problem 1.1 and Problem 1.2 under the assumption that (2.15) and (2.17) hold for some $\hat{Y}, \tilde{Y}$, and $H_{M_{2}}$. In general to find such $\hat{Y}, \tilde{Y}$, and $H_{M_{2}}$, we have to solve two matrix equations with three unknowns after substituting $(2.20),(2.22)$, and (2.23) into (2.15) and (2.17). 
To end this section, we consider a sufficient condition that

$$
K_{0} X_{1}=M_{0} X_{1} \Lambda, K_{1}=0, M_{1}=0,
$$

under which $(2.2)$ is reduced to

$$
\left(\begin{array}{cc}
K_{0} & 0 \\
0 & K_{2}
\end{array}\right)\left(\begin{array}{c}
X_{1} \\
X_{2}
\end{array}\right)=\left(\begin{array}{cc}
M_{0} & 0 \\
0 & M_{2}
\end{array}\right)\left(\begin{array}{c}
X_{1} \\
X_{2}
\end{array}\right) \Lambda
$$

i.e.,

$$
K_{2} X_{2}=M_{2} X_{2} \Lambda
$$

To solve (2.29) for symmetric matrices $K_{2}$ and $M_{2}$ is the symmetric inverse eigenvalue problem without constraints. Dai [7] studied this problem and gave an efficient algorithm for it by using QR-like decomposition with column pivoting and least squares techniques. Here we present the expression of the general symmetric solution only by generalized inverse and SVD.

TheOREM 2.8. Suppose $X_{2}$ and $\Lambda$ are known and the singular value decomposition of $X_{2}$ is (2.5). Then (2.29) has a symmetric solution and

$$
\left(K_{2}, M_{2}\right)=U\left(\begin{array}{cccc}
M_{11}^{(2)} \Sigma \Lambda_{11}^{(2)} \Sigma^{-1} & \left(M_{21}^{(2)} \Sigma \Lambda_{11}^{(2)} \Sigma^{-1}\right)^{T} & M_{11}^{(2)} & \left(M_{21}^{(2)}\right)^{T} \\
M_{21}^{(2)} \Sigma \Lambda_{11}^{(2)} \Sigma^{-1} & K_{22}^{(2)} & M_{21}^{(2)} & M_{22}^{(2)}
\end{array}\right) \operatorname{diag}(U, U)^{T}
$$

where $M_{11}^{(2)}=P_{Y}^{\perp} H P_{Y}^{\perp}$ with $H$ being a symmetric matrix such that $P_{Y}^{\perp} H P_{Y}^{\perp} \Sigma \Lambda_{11}^{(2)} \Sigma^{-1}$ is symmetric, $Y=\Sigma \Lambda_{12}^{(2)}, M_{21}^{(2)}=F P_{Y}^{\perp}$ with $F$ being arbitrary, $K_{22}^{(2)}, M_{22}^{(2)}$ are two arbitrary symmetric matrices, $\Lambda_{11}^{(2)}=V_{1}^{T} \Lambda V_{1}, \Lambda_{12}^{(2)}=V_{1}^{T} \Lambda V_{2}$.

Proof. Substitute (2.5) into (2.29).

Before we consider Problem 1.2 under the condition (2.28), we give some notation.

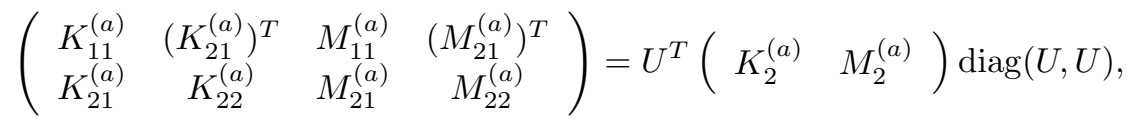

$$
\begin{aligned}
& \tilde{\Lambda}_{11}=\left(\begin{array}{cc}
\Sigma \Lambda_{11}^{(2)} \Sigma^{-1} & I
\end{array}\right), \widetilde{K M}_{11}=\left(\begin{array}{cc}
K_{11}^{(a)} & M_{11}^{(a)}
\end{array}\right), \widetilde{K M}_{21}=\left(\begin{array}{cc}
K_{21}^{(a)} & M_{21}^{(a)}
\end{array}\right) .
\end{aligned}
$$

Theorem 2.9. If $K_{0} X_{1}=M_{0} X_{1} \Lambda$ and $K_{1}=0, M_{1}=0$, Problem 1.2 has a solution and

$$
\left(K_{2}, M_{2}\right)=U\left(\begin{array}{cccc}
M_{11}^{(2)} \Sigma \Lambda_{11}^{(2)} \Sigma^{-1} & \left(M_{21}^{(2)} \Sigma \Lambda_{11}^{(2)} \Sigma^{-1}\right)^{T} & M_{11}^{(2)} & \left(F P_{Y}^{\perp}\right)^{T} \\
M_{21}^{(2)} \Sigma \Lambda_{11}^{(2)} \Sigma^{-1} & K_{22}^{(a)} & F P_{Y}^{\perp} & M_{22}^{(a)}
\end{array}\right) \operatorname{diag}(U, U)^{T},
$$


in which $M_{11}^{(2)}=P_{Y}^{\perp} H P_{Y}^{\perp}$ with $H$ being a symmetric solution of $\| P_{Y}^{\perp} H P_{Y}^{\perp} \tilde{\Lambda}_{11}-$ $\widetilde{K M}_{11} \|=\min ; P_{Y}^{\perp} H P_{Y}^{\perp} \Sigma \Lambda_{11}^{(2)} \Sigma^{-1}=\Sigma^{-1}\left(\Lambda_{11}^{(2)}\right)^{T} \Sigma P_{Y}^{\perp} H P_{Y}^{\perp} ;$ and $F=\widetilde{K M}_{21}\left(P_{Y}^{\perp} \tilde{\Lambda}_{11}\right)^{\dagger}$ $+W P_{P_{Y}^{\frac{1}{Y}} \tilde{\Lambda}_{11}}^{\perp}$ with $W$ being arbitrary.

Proof. It is clear that $\left\|K-K_{a}\right\|^{2}+\left\|M-M_{a}\right\|^{2}=\min$ if and only if

$$
\left\|K_{2}-K_{2}^{(a)}\right\|_{F}^{2}+\left\|M_{2}-M_{2}^{(a)}\right\|_{F}^{2}=\min .
$$

Equation (2.30) holds if and only if

$$
\begin{array}{ll}
\left\|M_{11}^{(2)} \tilde{\Lambda}_{11}-\widetilde{K M}_{11}\right\|^{2}=\min , & \left\|M_{21}^{(2)} \tilde{\Lambda}_{11}-\widetilde{K M}_{21}\right\|^{2}=\min , \\
\left\|K_{22}^{(2)}-K_{22}^{(a)}\right\|_{F}^{2}=\min , \quad\left\|M_{22}^{(2)}-M_{22}^{(a)}\right\|_{F}^{2}=\min .
\end{array}
$$

REMARK 2.10. For details about solving $\min _{H \in \mathbb{S R}^{n \times n}}\left\|P_{Y}^{\perp} H P_{Y}^{\perp} \tilde{\Lambda}_{11}-\widetilde{K M}_{11}\right\|$, see Theorem 3.3.2 in [18].

3. A special case. In this section, we consider one simple but not easy case of Problem 1.1 and Problem 1.2.

Problem 3.1. Given $p$ eigenpairs by $(X, \Lambda)$, where $X \in \mathbb{R}^{n \times p}$ and $\Lambda \in \mathbb{R}^{p \times p}$ is a block diagonal matrix with 2-by-2 blocks or single real eigenvalues on its main diagonal, and $K_{0} \in \mathbb{S R}^{r \times r}$. Find a real matrix $K \in \mathbb{S R}^{n \times n}$ such that

$$
K X=X \Lambda, K([1, r])=K_{0},
$$

where $K([1, r])$ is the $r \times r$ leading principal submatrix of $K$.

Problem 3.2. Given $K_{a} \in \mathbb{S R}^{n \times n}$ with $K_{a}([1, r])=K_{0}$, find $\hat{K} \in S_{E}$ such that

$$
\left\|K_{a}-\hat{K}\right\|=\inf _{K \in S_{E}}\left\|K_{a}-K\right\|,
$$

where $S_{E}$ is the solution set of Problem 3.1.

Let $X \in \mathbb{R}^{n \times p}$ and $K \in \mathbb{S R}^{n \times n}$ have the partitions as in (2.1) and the singular value decomposition of $X_{2}$ be given as in (2.5).

TheOREm 3.3. Suppose that $K_{0} \in \mathbb{S R}^{r \times r}, X \in \mathbb{R}^{n \times p}$, and $\Lambda \in \mathbb{R}^{p \times p}$ are given as in Problem 3.1. Then Problem 3.1 is solvable if and only if

$$
\left(K_{0} X_{1}-X_{1} \Lambda\right) P_{X_{2}^{T}}^{\perp}=0, \quad P_{X_{2}^{T}}^{\perp}\left(\Lambda^{T} X_{2}^{T}-X_{1}^{T}\left(X_{1} \Lambda-K_{0} X_{1}\right) X_{2}^{\dagger}\right)=0,
$$

and

$$
X^{T} X \Lambda=\Lambda^{T} X^{T} X
$$

hold. 
If (3.2) and (3.3) hold, then the general solution of Problem 3.1 is

$$
K=\left(\begin{array}{cc}
K_{0} & \left(X_{1} \Lambda-K_{0} X_{1}\right) X_{2}^{\dagger}+P_{\left(X_{1} P_{X_{2}^{T}}^{\perp}\right)}^{\perp} L P_{X_{2}}^{\perp} \\
K_{1}^{T} & B X_{2}^{\dagger}+\left(B X_{2}^{\dagger}\right)^{T} P_{X_{2}}^{\perp}+P_{X_{2}}^{\perp} H P_{X_{2}}^{\perp}
\end{array}\right),
$$

where $B=X_{2} \Lambda-\left(X_{2}^{\dagger}\right)^{T}\left(X_{1} \Lambda-K_{0} X_{1}\right)^{T} X_{1}-P_{X_{2}}^{\perp} L^{T} P_{\left(X_{1} P_{X_{2}^{T}}^{\perp}\right)}^{\perp} X_{1}$, both $L \in \mathbb{R}^{r \times(n-r)}$ and $H \in \mathbb{S R}^{(n-r) \times(n-r)}$ are arbitrary.

Proof. Applying (2.1), (3.1) is equivalent to the following two equations

$$
\begin{aligned}
K_{1} X_{2} & =X_{1} \Lambda-K_{0} X_{1}, \\
K_{1}^{T} X_{1} & =X_{2} \Lambda-K_{2} X_{2} .
\end{aligned}
$$

By Lemma 2.2, (3.5) has a solution $K_{1}$ if and only if

$$
\left(X_{1} \Lambda-K_{0} X_{1}\right) P_{X_{2}^{T}}^{\perp}=0,
$$

in which case,

$$
K_{1}=\left(X_{1} \Lambda-K_{0} X_{1}\right) X_{2}^{\dagger}+Y P_{X_{2}}^{\perp},
$$

where $Y \in \mathbb{R}^{r \times(n-r)}$ is arbitrary. Substitute (3.7) into (3.6),

(3.8) $K_{2} X_{2}=X_{2} \Lambda-K_{1}^{T} X_{1}=X_{2} \Lambda-\left(X_{2}^{\dagger}\right)^{T}\left(X_{1} \Lambda-K_{0} X_{1}\right)^{T} X_{1}-P_{X_{2}}^{\perp} Y^{T} X_{1}$.

By Lemma 2.1, there exists a symmetric matrix $K_{2}$ satisfying (3.8) if and only if

$$
\begin{aligned}
\left(X_{2} \Lambda-K_{1}^{T} X_{1}\right) P_{X_{2}^{T}}^{\perp} & =0, \\
P_{X_{2}}\left(X_{2} \Lambda-K_{1}^{T} X_{1}\right) X_{2}^{\dagger} & \in \mathbb{S R}^{q \times(n-r)} .
\end{aligned}
$$

Let $G=P_{X_{2}^{T}}^{\perp}\left(\Lambda^{T} X_{2}^{T}-X_{1}^{T}\left(X_{1} \Lambda-K_{0} X_{1}\right) X_{2}^{\dagger}\right)$. Since $P_{X_{2}^{T}}^{\perp}\left(P_{X_{2}^{T}} X_{1}^{T}\right)\left(P_{X_{2}^{T}} X_{1}^{T}\right)^{\dagger}=0$,

$$
P_{X_{2}^{T}} X_{1}^{T}\left(P_{X_{2}^{T}} X_{1}^{T}\right)^{\dagger} G\left(P_{X_{2}}^{\perp}\right)^{\dagger} P_{X_{2}}^{\perp} \equiv 0 .
$$

Substitute (3.7) into (3.9), to get

$$
P_{X_{2}^{T}}^{\perp} X_{1}^{T} Y P_{X_{2}}^{\perp}=G
$$

By (3.11) and Lemma 2.2, (3.12) holds if and only if $G=0$, which is exactly the second equation in (3.2), and in this case

$$
Y=L-\left(P_{X_{2}^{T}}^{\perp} X_{1}^{T}\right)^{\dagger}\left(P_{X_{2}^{T}}^{\perp} X_{1}^{T}\right) L P_{X_{2}}^{\perp},
$$

where $L \in \mathbb{R}^{r \times(n-r)}$ is arbitrary. Substituting (3.13) into (3.7), we have

$$
K_{1}=\left(X_{1} \Lambda-K_{0} X_{1}\right) X_{2}^{\dagger}+P_{\left(X_{1} P_{X_{2}^{T}}^{\perp}\right)}^{\perp} L P_{X_{2}}^{\perp} .
$$


Now we substitute (3.14) into (3.10), to get

$$
\begin{aligned}
P_{X_{2}}\left(X_{2} \Lambda-K_{1}^{T} X_{1}\right) X_{2}^{\dagger} & =\left(X_{2} \Lambda-X_{2} X_{2}^{\dagger} K_{1}^{T} X_{1}\right) X_{2}^{\dagger} \\
& =X_{2} \Lambda X_{2}^{\dagger}-\left(X_{2}^{\dagger}\right)^{T} \Lambda^{T} X_{1}^{T} X_{1} X_{2}^{\dagger}+\left(X_{1} X_{2}^{\dagger}\right)^{T} K_{0}^{T} X_{1} X_{2}^{\dagger} \\
& =\left(X_{2}^{\dagger}\right)^{T}\left(X_{2}^{T} X_{2} \Lambda-\Lambda^{T} X_{1}^{T} X_{1}\right) X_{2}^{\dagger}+\left(X_{1} X_{2}^{\dagger}\right)^{T} K_{0} X_{1} X_{2}^{\dagger} .
\end{aligned}
$$

So we can see that the condition (3.10) is equivalent to (3.3). At this point, we have found the necessary and sufficient condition for the solvability of Problem 3.1. Next we prove that (3.4) is the general solution of Problem 3.1 if (3.2) and (3.3) hold. If (3.2) and (3.3) hold, we have proved that $K_{1}$ has the expression as in (3.14); what is left to prove is $K_{2}=B X_{2}^{\dagger}+\left(B X_{2}^{\dagger}\right)^{T} P_{X_{2}}^{\perp}+P_{X_{2}}^{\perp} H P_{X_{2}}^{\perp}$. Indeed, if (3.2) and (3.3) hold, then (3.9) and (3.10) are satisfied. Applying Lemma 2.1 and substituting (3.13) into (3.8), (3.8) has a symmetric solution $K_{2}=B X_{2}^{\dagger}+\left(B X_{2}^{\dagger}\right)^{T} P_{X_{2}}^{\perp}+P_{X_{2}}^{\perp} H P_{X_{2}}^{\perp}$.

If $X_{2}$ is square and nonsingular, then $P_{X_{2}}^{\perp}=0$ and $P_{X_{2}^{T}}^{\perp}=0$ and $B=X_{2} \Lambda-$ $\left(X_{2}^{-1}\right)^{T}\left(X_{1} \Lambda-K_{0} X_{1}\right)^{T} X_{1}$. So we have

Corollary 3.4. Suppose that $K_{0} \in \mathbb{S R}^{r \times r}, X \in \mathbb{R}^{n \times p}$, and $\Lambda \in \mathbb{R}^{p \times p}$ is a block diagonal matrix. If $X_{2}$ is square and nonsingular, then Problem 3.1 has a unique solution

$$
K=\left(\begin{array}{cc}
K_{0} & \left(X_{1} \Lambda-K_{0} X_{1}\right) X_{2}^{-1} \\
K_{1}^{T} & X_{2} \Lambda X_{2}^{-1}-\left(X_{2}^{-1}\right)^{T}\left(X_{1} \Lambda-K_{0} X_{1}\right)^{T} X_{1} X_{2}^{-1}
\end{array}\right)
$$

if and only if $X^{T} X \Lambda=\Lambda X^{T} X$.

Now we present a general solution of Problem 3.2 assuming Problem 3.1 is solvable.

TheOREM 3.5. For given matrices $K_{a} \in \mathbb{R}^{n \times n}$ with $K_{a}([1, r])=K_{0}$ as in (2.25). If (3.2) and (3.3) hold, then Problem 3.2 has a unique solution

$$
\hat{K}=\left(\begin{array}{cc}
K_{0} & \left(X_{1} \Lambda-K_{0} X_{1}\right) X_{2}^{\dagger}+\tilde{L} \\
K_{1}^{T} & B X_{2}^{\dagger}+\left(B X_{2}^{\dagger}\right)^{T} P_{X_{2}}^{\perp}+P_{X_{2}}^{\perp} K_{2}^{(a)} P_{X_{2}}^{\perp}
\end{array}\right),
$$

where $B=X_{2} \Lambda X_{2}^{\dagger}-\left(X_{2}^{\dagger}\right)^{T}\left(X_{1} \Lambda-K_{0} X_{1}\right)^{T} X_{1} X_{2}^{\dagger}-\tilde{L}^{T} X_{1} X_{2}^{\dagger}, \widetilde{L}$ satisfies

$$
\begin{aligned}
\operatorname{vec}(\widetilde{L})= & {\left[\left(X_{1} X_{2}^{\dagger}\right)^{T} \otimes\left(X_{1} X_{2}^{\dagger}\right) Q+2 I \otimes\left(I+\left(X_{1} X_{2}^{\dagger}\right)\left(X_{1} X_{2}^{\dagger}\right)^{T}\right)\right]^{-1} } \\
& \operatorname{vec}\left(2 \widetilde{K_{1}}-\left(X_{1} X_{2}^{\dagger}\right)\left(\widetilde{K_{2}}+{\widetilde{K_{2}}}^{T}\right)\right),
\end{aligned}
$$

$Q$ is a permutation matrix such that $\operatorname{vec}\left(\widetilde{L}^{T}\right)=Q \operatorname{vec}(\widetilde{L})$ and $\tilde{K}_{1}=K_{1}^{(a)}-\left(X_{1} \Lambda-\right.$ $\left.K_{0} X_{1}\right) X_{2}^{\dagger}$.

Proof. Let $\widetilde{L}=P_{\left(X_{1} P_{X_{2}^{T}}^{\perp}\right)}^{\perp} L P_{X_{2}}^{\perp}$. From Theorem 3.3,

$$
\left\|K^{(a)}-K\right\|^{2}=2\left\|K_{1}^{(a)}-K_{1}\right\|^{2}+\left\|K_{2}^{(a)}-K_{2}\right\|^{2}
$$




$$
\begin{aligned}
= & 2\left\|K_{1}^{(a)}-\left(X_{1} \Lambda-K_{0} X_{1}\right) X_{2}^{\dagger}-\tilde{L}\right\|^{2} \\
& +\| K_{2}^{(a)}-X_{2} \Lambda X_{2}^{\dagger}+\left(X_{2}^{\dagger}\right)^{T}\left(X_{1} \Lambda-K_{0} X_{1}\right)^{T} X_{1} X_{2}^{\dagger} \\
& +\tilde{L}^{T} X_{1} X_{2}^{\dagger}+\left(X_{1} X_{2}^{\dagger}\right)^{T} \tilde{L}-P_{X_{2}}^{\perp} H P_{X_{2}}^{\perp} \|^{2} .
\end{aligned}
$$

By Lemma 2.5,

$$
\begin{aligned}
\min _{H=H^{T}}\left\|K^{(a)}-K\right\|^{2}= & 2\left\|K_{1}^{(a)}-\left(X_{1} \Lambda-K_{0} X_{1}\right) X_{2}^{\dagger}-\tilde{L}\right\|^{2} \\
& +\| K_{2}^{(a)}-X_{2} \Lambda X_{2}^{\dagger}+\left(X_{2}^{\dagger}\right)^{T}\left(X_{1} \Lambda-K_{0} X_{1}\right)^{T} X_{1} X_{2}^{\dagger} \\
& +\tilde{L}^{T} X_{1} X_{2}^{\dagger}+\left(X_{1} X_{2}^{\dagger}\right)^{T} \tilde{L}-P_{X_{2}}^{\perp} K_{2}^{(a)} P_{X_{2}}^{\perp} \|^{2},
\end{aligned}
$$

and the minimum is attained if and only if $P_{X_{2}}^{\perp}\left(H-K_{2}^{(a)}\right) P_{X_{2}}^{\perp}=0$. Let $\tilde{K}_{1}=K_{1}^{(a)}-$ $\left(X_{1} \Lambda-K_{0} X_{1}\right) X_{2}^{\dagger}, \tilde{K}_{2}=K_{2}^{(a)}-P_{X_{2}}^{\perp} K_{2}^{(a)} P_{X_{2}}^{\perp}-X_{2} \Lambda X_{2}^{\dagger}+\left(X_{2}^{\dagger}\right)^{T}\left(X_{1} \Lambda-K_{0} X_{1}\right)^{T} X_{1} X_{2}^{\dagger}$, and

$$
f(\tilde{L})=2\left\|\tilde{K}_{1}-\tilde{L}\right\|^{2}+\left\|\tilde{K}_{2}+\tilde{L}^{T} X_{1} X_{2}^{\dagger}+\left(X_{1} X_{2}^{\dagger}\right)^{T} \tilde{L}\right\|^{2} .
$$

Then

$$
\begin{aligned}
f(\tilde{L})= & 2 \operatorname{tr}\left(\tilde{K}_{1}^{T} \tilde{K}_{1}\right)+2 \operatorname{tr}\left(\tilde{L}^{T} \tilde{L}\right)+\operatorname{tr}\left(\tilde{K}_{2}^{T} \tilde{K}_{2}\right)+2 \operatorname{tr}\left(\tilde{K}_{2}^{T}\left(X_{1} X_{2}^{\dagger}\right)^{T} \tilde{L}\right) \\
& +2 \operatorname{tr}\left(\tilde{K}_{2}\left(X_{1} X_{2}^{\dagger}\right)^{T} \tilde{L}\right)+2 \operatorname{tr}\left(X_{1} X_{2}^{\dagger}\left(X_{1} X_{2}^{\dagger}\right)^{T} \tilde{L} \tilde{L}^{T}\right) \\
& +2 \operatorname{tr}\left(\left(X_{1} X_{2}^{\dagger}\right)^{T} \tilde{L}\left(X_{1} X_{2}^{\dagger}\right)^{T} \tilde{L}\right)-4 \operatorname{tr}\left(\tilde{K}_{1}^{T} \tilde{L}\right)
\end{aligned}
$$

Consequently, to get the minimal value of $f(\tilde{L})$, we should find its minimal point. As

$\frac{\partial f(\tilde{L})}{\partial \tilde{L}}=2\left(X_{1} X_{2}^{\dagger}\right) \tilde{L}^{T}\left(X_{1} X_{2}^{\dagger}\right)+4\left(I+\left(X_{1} X_{2}^{\dagger}\right)\left(X_{1} X_{2}^{\dagger}\right)^{T}\right) \tilde{L}-4 \tilde{K}_{1}+2\left(X_{1} X_{2}^{\dagger}\right)\left(\tilde{K}_{2}+\tilde{K}_{2}^{T}\right)$,

$\frac{\partial f(\tilde{L})}{\partial \tilde{L}}=0$ is equivalent to

$$
\Phi \operatorname{vec}(\tilde{L})=\operatorname{vec}\left(2 \tilde{K}_{1}-\left(X_{1} X_{2}^{\dagger}\right)\left(\tilde{K}_{2}+\tilde{K}_{2}^{T}\right)\right),
$$

where $\Phi=\left(X_{1} X_{2}^{\dagger}\right)^{T} \otimes\left(X_{1} X_{2}^{\dagger}\right) Q+2 I \otimes\left(I+\left(X_{1} X_{2}^{\dagger}\right)\left(X_{1} X_{2}^{\dagger}\right)^{T}\right)$, and $Q$ is a permutation matrix such that $\operatorname{vec}\left(\tilde{L}^{T}\right)=Q \operatorname{vec}(\tilde{L})$. Since $\Phi$ is nonsingular, (3.16) has an unique solution $(3.15)$ and $f(\tilde{L})$ has an unique minimal value point $\tilde{L}$. $\square$

4. Conclusions. Motivated by Y. Yuan and H. Dai [21], this paper is concerned with the symmetric inverse generalized eigenvalue problem and the problem of the optimal approximation for a given symmetric matrix pencil under a given spectral constraint and a symmetric submatrix pencil constraint. By using the Moore-Penrose generalized inverse and the singular value decomposition, we first present the solvability condition and the expression for the solution of these two problems. For the 
case that the mass matrix is unit, there is a formula of the general solution which is inexpensive to compute and can be used routinely in practice.

Acknowledgment. The authors are grateful to the editor and the anonymous referee for their useful comments and suggestions, which greatly improved the original presentation.

\section{REFERENCES}

[1] M. Baruch. Optimization procedure to correct stiffness and flexibility matrices using cibration tests. AIAA Journal, 16:1208-1210, 1978.

[2] M. Baruch. Optimal correction of mass and stiffness matrices using measured modes. AIAA Journal, 20:1623-1626, 1982.

[3] A. Ben-Israel and T.N.E. Greville. Generalized Inverses: Theory and Applications, 2nd edition. Springer-Verlag, New York, 2003.

[4] A. Berman and W.G. Flannely. Theory of incomplete models of dynamic structures. AIAA Journal, 9:1481-1487, 1971.

[5] R.G. Cobb and B.S. Liebst. Structural damage identification using assigned partial eigenstructure. AIAA Journal, 35:152-158, 1997.

[6] H. Dai. About an inverse eigenvalue problem arising in vibration analysis. RAIRO Modélisation Mathématique et Analyse Numérique 29:421-434, 1995.

[7] H. Dai. An algorithm for symmetric generalized inverse eigenvalue problems. Linear Algebra and its Applications, 296:79-98, 1999.

[8] W.L. Li. A new method for structural model updating and joint stiffness identification. Mechanical Systems and Signal Processing, 16:155-167, 2002.

[9] M. Link. Identification and correction of errors in analytical models using test data-theoretical and practical bounds. The Proceedings of the 8th International Modal Analysis Conference, Florida, USA, 570-578, 1990.

[10] W. Luber and A. Lotze. Application of sensitivity methods for error localization in finite element systems. The Proceedings of the 8th International Modal Analysis Conference, Florida, USA, 598-604, 1990.

[11] C. Minas and D.J. Inman. Matching finite element models to modal data. Journal of Vibration and Acoustics, 112:84-92, 1990.

[12] J.E. Mottershead and M.I. Friswell. Model updating in structural dynamics: a survey. Journal of Sound and Vibration, 167:347-375, 1993.

[13] J.C. O'Callahan and C.M. Chou. Localization of model errors in optimized mass and stiffness matrices using modal-test data. International Journal of Analytical and Experimental Analysis, 4:8-14, 1989.

[14] F. Tisseur. A chart of backward errors for singly and doubly structured eigenvalue problems. SIAM Journal on Matrix Analysis and Applications, 24:877-897, 2003.

[15] W.F. Trench. Inverse eigenproblems and associated approximation problems for matrices with generalized symmetry or skew symmetry. Linear Algebra and its Applications, 380:199-211, 2004.

[16] F.S. Wei. Mass and stiffness interaction effects in analytical model modification. AIAA Journal, 28:1686-1688, 1990.

[17] F.S. Wei and D.W. Zhang. Mass matrix modification using element correction method. AIAA Journal, 27:119-121, 1989.

[18] M. Wei. Theory and Calculation of Generalized Least Square Problem. Science Press, Beijing, 
2006 (in Chinese).

[19] H.Q. Xie. Sensitivity Analysis of Eigenvalue Problem. Ph.D. Dissertation, Nanjin University of Aeronautics and Astronautica, 2003.

[20] Y. Yuan and H. Dai. Inverse problems for symmetric matrices with a submatrix constraint. Applied Numerical Mathematics, 57:646-656, 2007.

[21] Y. Yuan and H. Dai. A generalized inverse eigenvalue problem in structural dynamic model updating. Journal of Computational and Applied Mathematics, 226:42-49, 2009.

[22] O. Zhang, A. Zerva, and D.W. Zhang. Stiffness matrix adjustment using incomplete measured modes. AIAA Journal, 35:917-919, 1997. 